\title{
CRITICAL STUDY OF THE BURSTING STRENGTH TEST FOR PAPER
}

\author{
By F. T. Carson and F. V. Worthington
}

\section{ABSTRACT}

An extensive investigation has been made of alleged variables in the bursting test. The study included clamping pressure, nature of clamping surfaces, size of bursting orifice, types of diaphragms, effect of manner of installation of diaphragms, effect of air entrapped under diaphragm, speed of operation, effect of viscosity of liquid medium in hydraulic chamber, calibration and performance of pressure gauges, relation of bursting to tensile results, and relation of size of bursting orifice to bursting pressure. A device is described for measuring the vertical deflection of the paper in the bursting test.

As a result of the investigation it is recommended that a clamping pressure be used corresponding to a total load on the specimen of approximately 1,000 pounds. An all-metal clamping head having a relatively smooth clamping surface and a bursting orifice 1.20 inches in diameter is recommended to replace the rubber-faced clamping head formerly used. No evidence was found that the type of diaphragms ordinarily used, the manner of installation, or the viscosity of the hydraulic medium used have a significant effect upon the test. The results are affected to some extent by the testing rate, being somewhat greater the higher the testing rate. Air in the hydraulic chamber, because of its compressibility, has the effect of reducing the testing rate. The testing rate may conveniently be expressed in terms of the time interval during which the specimen is being stressed. For the official method this corresponds to about 1 second. Because of the difficulty of properly adjusting the pressure gauges of the type commonly used on the bursting tester for operation at high speed, the official testing rate is about the maximum that could safely be used. The equation $P R=2 T$, in which $P$ is the bursting pressure, $R$ the radius of curvature of the paper at rupture in the bursting test, and $T$ the machine direction tensile breaking strength per unit width, relates the bursting and tensile properties and enables a correlation to be made of data obtained with bursting orifices of different sizes.

\section{CONTENTS}

I. Introduction

II. Testing procedure

III. Variables investigated

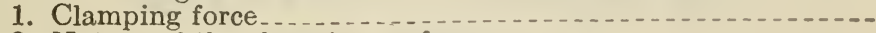

2. Nature of the clamping surfaces

3. Testing rate $\ldots \ldots$

4. Diaphragm error $\ldots \ldots$

5. Effect of the viscosity of the liquid ........................

6. Operation of pressure gauges and their calibration.........

7. Size of the orifice in the clamping head

IV. Comparison of the new, motor-driven testers with the older, hand-

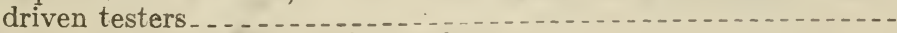

V. Relation of bursting to tensile results

1. Theory . .

2. Special apparatus required .

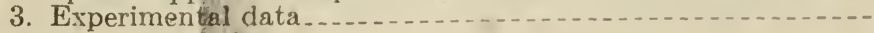

VI. Conclusions 


\section{INTRODUCTION}

The bursting strength test is one of the oldest and most widely used criteria of the strength of paper. A sort of empirical, composite test, combining the effects of both the tensile strength and the elongation of paper, it is generally considered to be a fairly dependable means of determining the relative strength of papers in relation to many ordinary uses. The test is quickly and easily made by means of a simple, sturdy testing device, one form of which is shown in Figure 1. Nearly all specifications for paper include the bursting test, and in some cases this is the only means specified for strength measurement.

The device for making the bursting test is provided with a platform containing a central circular orifice over which is placed the sheet of paper to be tested. A clamping head is brought down on top of the paper with sufficient pressure to hold it in place. In this clamping head there is an orifice which registers with the orifice in the platform. A piston, driven by hand or by motor, forces liquid up through the orifice in the platform and distends the test specimen through the orifice in the clamping head until the paper burst. The pressure in the hydraulic chamber at the instant of rupture is indicated on a maximum-reading pressure gauge of the Bourdon tube type. A rubber diaphragm covers the lower orifice to prevent liquid from coming. in direci contact with the paper being tested.

\section{TESTING PROCEDURE}

In studying the performance of a testing device it would obviously be desirable to have available for comparative tests a material of such uniformity that a few tests made while changing one variable at a time would reveal the characteristics of the tester without the uncertainty due to the variability of the material tested. In the study of a paper testing device one is handicapped at the outset by the lack of uniformity of paper and the consequent variability of the test results. In the subsequent tables most of the tabulated results are averages of 20 tests of representative samples. The various papers employed are designated in the tables by their trade names. All tests were made at 65 per cent relative humidity and $70^{\circ} \mathrm{F}$. after the test specimens had been conditioned to equilibrium in this atmosphere.

\section{VARIABLES INVESTIGATED}

\section{CLAMPING FORCE}

The specimen must not slip during the test, for otherwise the indicated bursting strength will be too high. Insufficient clamping force is probably responsible for many discrepancies in bursting tests.

Within certain limits the resistance to slipping is practically independent of the area of the gripping surfaces, provided the total load on the clamp is kept constant. Slipping can not, therefore, be overcome by a change in the area of the clamping surfaces, but must be corrected by increasing the total force or load which is applied to the clamp. In the bursting tester shown in Figure 1, the clamping 
force may be approximately evaluated by calibrating the clamping spring. The clamping force necessary to prevent slipping of the specimen was measured for five different kinds of paper chosen so as to be representative of the range of bursting pressures ordinarily encountered, and was found to range from 350 to $\$ 00$ pounds, depending upon the character of the paper. It appears, therefore, that a clamping force of about 1,000 pounds is necessary to insure against slipping in all cases. This corresponds to a compression of the usual clamping spring of about a quarter of an inch. The operator should watch carefully at all times for any movement of the unclamped margin of the specimen. If slipping is indicated the result should of course be discarded and the clamping pressure increased.

\section{NATURE OF CLAMPING SURFACES}

For many years the bursting tester has been equipped with a rubber ring which served as the clamping surface and bursting orifice in the clamping head. From comparative tests of clamping heads, Snyder ${ }^{1}$ concluded that the all-metal clamp is superior in gripping efficiency to the rubber ring type. This conclusion has been questioned by Abrams. ${ }^{2}$ The experiments on clamping pressure made in the course of the present investigation showed that a greater clamping force is required to prevent a given paper from slipping when the rubber ring type of clamping head is used than when the all-metal clamping head is used. Hence, it appears that the differences in test results noted by Snyder are attributable in part to the size of the orifice (Sec. III, 7) and in part to the greater gripping efficiency of the metal clamping surface.

Because of this superior gripping efficiency of the metal clamping surface and because of its permanence in contrast with the wellknown tendency of the rubber ring to deteriorate with age, it has been definitely recommended that the rubber ring type of clamping head be replaced with the all-metal construction, and the recommendation has been followed by the manufacturer of the instrument.

When this recommendation was being discussed consideration was given to the possible shearing effect of the relatively sharp edge of the clamping surface bounding the bursting orifice. This apparent difficulty can be dismissed, however, since paper does not normally break at the edge of the orifice. Paper breaks more as a result of the slipping of fibers than of the rupture of fibers, and, hence, the region about the edge of the orifice where the fibers are held by clamping pressure would normally be the last place where a rupture would begin. As a matter of experimental fact the rupture almost never originates in this region. Should the edge of the clamp be sharp enough to cut the paper it would be immediately recognized by an abnormal rupture originating at the edge of the orifice. On the other hand, if this edge were excessively rounded, the consequence would be an increase of the effective orifice diameter, the effect of which can be inferred from the discussion in Section III, 7. This edge of the clamping surface on the clamping heads now being fur-

1 Leo W. Snyder, Effect of the Clamping Device of the Mullen Paper Tester on Test Results, Paper Trade Jour.; Feb. 12, 1925, and A Study of the Mullen Paper Tester, Paper Trade Jour.; Aug. 4, 1927.

Allen Abrams, A study of the Mullen Tester on Paper Board, Paper Trade Jour.; Aug. 6, 1925.

$30894^{\circ}-31-11$ 
nished is merely relieved of sharpness without being appreciably rounded, and the construction appears to be quite uniform for such as have been examined.

\section{TESTING RATE}

As with tensile tests of paper, the bursting strength depends slightly upon the rate of applying the load. The officially designated testing rate is 120 revolutions of the hand wheel per minute. The results given in Table 1 (gauge $\mathrm{C}$ ) show that doubling the testing rate (that is, halving the stressing interval) increases the bursting pressure on the average by about 4 per cent.

Air in the hydraulic chamber, since it is compressible, has the effect of reducing the testing rate. Ordinarily a speed of 120 revolutions per minute will cause a paper bursting at about 50 or 60 pounds per square inch to break in about 1 second after the pressure begins to build up. The introduction of a large air bubble (about $1 \mathrm{~cm}^{3}$ ), into the hydraulic chamber will about double this time interval. Reed and Veitch ${ }^{3}$ give a simple method for inspecting the tester for entrapped air. Its presence in quantity is also made evident by the relatively loud sound when paper bursts.

The maximum-reading pressure gauge commonly used with the bursting tester is equipped with a damping spring which bears against the pointer shaft and is intended to hold the pointer in the position attained at the instant the paper gives way. Insufficient friction of this spring on the shaft tends to make the pointer register too high because of the momentum of the moving parts and too much friction causes the movement to be sluggish and irregular. As the testing rate is increased the adjustment becomes more and more critical.

A series of tests was made at different testing rates with three gauges attached to the tester. Gauge $\mathrm{A}$ had friction enough to make the response sluggish and jerky. Gauge B had very light friction. Gauge $\mathrm{C}$ was given the optimum friction adjustment; that is, the maximum friction which would not result in sluggish or irregular response to pressure change. All three gauges were carefully calibrated. Three testing rates were used, the normal rate which corresponds to a stressing interval of 1 second, and stressing intervals, respectively, one-half and twice normal. The results are given in Table 1. The poorly adjusted gauges show greater error the higher the testing rate and the greater the gauge reading. There appears to be no particular difficulty in adjusting a gauge so that it will behave properly for the normal testing rate. But when this rate is doubled it is very difficult to adjust the gauge so that it will neither lag nor overshoot. For this reason alone, testing rates higher than that prescribed in the official method (corresponding to a stressing interval of approximately 1 second for paper bursting at 50 to 75 pounds per square inch) should not be used. Probably the erratic results occasionally reported and ascribed to the effect of testing rate are primarily due to the use of an improperly adjusted gauge.

8 E. O. Reed and F. P. Veitch, The Bursting Strength of Paper, Paper Trade J.; July 20, 1922. 
TABLE 1.-Effect of testing rate upon bursting tests

\begin{tabular}{|c|c|c|c|c|c|c|c|c|c|}
\hline \multirow{3}{*}{ Paper } & \multicolumn{9}{|c|}{ Bursting pressure } \\
\hline & \multicolumn{3}{|c|}{ 1/2-second stressing interval } & \multicolumn{3}{|c|}{ 1-second stressing interval } & \multicolumn{3}{|c|}{ 2-second stressing interval } \\
\hline & Gauge A & Gauge B & Gauge $\mathrm{C}$ & Gauge A & Gauge B & Gauge C & Gauge A & Gauge $B$ & Gauge C \\
\hline Bond & $\begin{array}{c}\text { Lbs./in. }{ }^{2} \\
39.8\end{array}$ & $\begin{array}{c}L b s . / \text { in }^{2}{ }^{2} \\
41.6\end{array}$ & $\begin{array}{c}\text { Lbs./in. }{ }^{2} \\
39.8\end{array}$ & $\begin{array}{c}\text { Lbs./in }{ }^{2} \\
38.6\end{array}$ & $\begin{array}{c}\text { Lbs./in }^{2}{ }^{2} \\
39.0\end{array}$ & $\begin{array}{c}\text { Lbs./in . } \\
38.2\end{array}$ & $\begin{array}{c}\text { Lbs./in. }{ }^{2} \\
37.0\end{array}$ & $\begin{array}{c}\text { Lbs./in. }{ }^{2} \\
37.5\end{array}$ & $\begin{array}{r}\text { Lbs./in. } \\
36.8\end{array}$ \\
\hline test) & 39.0 & 40.1 & 38.9 & 39.3 & 40.0 & 39.0 & 36.8 & $37 . €$ & \\
\hline $\begin{array}{l}\text { Ledger } \\
\text { Ledger (duplicate }\end{array}$ & 56.0 & 58.3 & 56.8 & 54.7 & 56.5 & 55.5 & 50.7 & 50.9 & 50.3 \\
\hline test) & 56.1 & 58. 0 & 57.2 & 54.1 & 55.6 & 54.5 & 52.5 & 53.9 & 52.7 \\
\hline
\end{tabular}

Nоте.-The friction adjustment of the gauges was as follows: Gauge A, considerable friction; gauge B, light friction; gauge $\mathrm{C}$, good adjustment.

\section{DIAPHRAGM ERROR}

The type of diaphragm ordinarily used as a separator between the glycerine chamber and the specimen is a piece of sheet rubber about 0.03 inch in thickness. A small fraction of the pressure applied in bursting the paper is required to distend the rubber diaphragm, this pressure increment being known as the diaphragm error. When the diaphragm is clamped in place it tends to give toward the center to form a pocket which when it is inverted by the inflowing glycerino may be raised 0.1 to 0.2 inch above the plane of the clamping surfaces before the rubber begins to stretch. Since the elongation at rupture of paper in the machine direction is seldom greater than about 5 per cent, which corresponds to a rise of the paper of about 0.17 inch in the bursting test, the diaphragm error can not be very significant in any case and is, perhaps, wholly negligible in most cases. The least pocket effect is produced when the diaphragn is installed dry. It can be considerably increased by wetting the diaphragm near the edge with glycerine before it is fastened in place. In Table 2 are shown the results obtained after installing a diaphragm wet with glycerine near the edge as compared with a dry diaphragm instillation. The manner of installing the diaphragm has a negligible effect on the test results with ordinary papers. It is possible that the aging of the rubber might increase the resistance to distention an appreciable amount. If, however, the diaphragm is installed with an ample pocket effect and is supple to the feel there is no apparent reason why it should give any trouble. The above observations do not apply to those heavy-duty testers having a considerably greater diaphragm error, nor to tests of materials which stretch considerably.

TABLE 2.-Effect of manner of installing diaphragm

\begin{tabular}{|c|c|c|}
\hline \multirow{2}{*}{ Paper } & \multicolumn{2}{|c|}{$\begin{array}{l}\text { Bursting pressure, } \\
\text { diaphragm installed }\end{array}$} \\
\hline & Wet & Dry \\
\hline $\begin{array}{l}\text { Writing } \\
\text { Bond_.......... } \\
\text { Ledger..... }\end{array}$ & $\begin{array}{c}\text { Lbs./in. }{ }^{2} \\
17.6 \\
39.1 \\
56.7\end{array}$ & $\begin{array}{c}\text { Lb8./in.2 } \\
17.6 \\
39.2 \\
57.2\end{array}$ \\
\hline
\end{tabular}




\section{EFFECT OF THE VISCOSITY OF THE LIQUID}

The liquid used in the hydraulic chamber of the bursting tester is glycerine, which is rather viscous, especially at low temperatures. Pressure is built up in this medium at a rather rapid rate in making a bursting test. Since the Bourdon tube often contains more or less air, which is compressible, it is necessary to force some glycerine at a considerable velocity through the small opening leading to the Bourdon tube in order for the gauge to register. It is therefore not unreasonable to expect the gauge reading to lag somewhat behind the true pressure value within the tester. This lag should be a function of the viscosity of the pressure medium. The reality of such a time lag was demonstrated experimentally, but it was found to be very small. Since the time required to build up the rupturing pressure in the tester is also small, tests of paper were made with liquids of different viscosities in order to determine the significance of this lag. The results are shown in Table 3. From these results it may be concluded that the use of glycerine in the tester instead of a more fluid medium can not be regarded as a source of sensible error.

TABLE 3.-Effect of the viscosity of the liquid used in the hydraulic chamber of the bursting tester

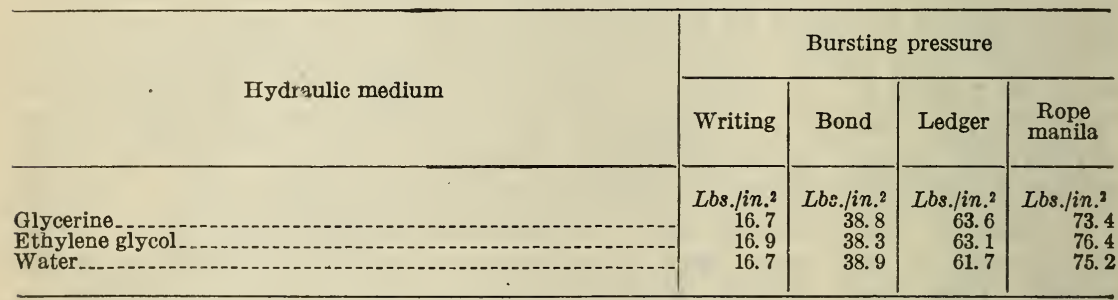

\section{OPERATION OF PRESSURE GAUGES AND THEIR CALIBRATION}

Because of its relatively delicate construction the gauge requires more in the way of care, adjustment, and calibration than does the bursting tester itself. The operating conditions are rather severe. The pressure is built up rapidly and released suddenly, conditions which are not favorable to permanence of calibration. As a consequence, frequent calibrations are essential.

A common deficiency in the operation of the bursting tester gauge is a sluggish or irregular response of the pointer to a change in pressure. A good way to inspect the gauge for such trouble is to clamp a piece of sheet metal in the tester, turn the handwheel up until the gauge begins to register pressure, and then to continue the application of pressure by small steps. The gauge hand should respond immediately to each small pressure change. If the response is sluggish or irregular the difficulty is probably due to foreign matter in the teeth of the sector and pinion or to excessive friction of the damping spring on the pinion shaft. Mechanical deficiencies should be remedied before the gauge is calibrated. The screws fastening the gauge mechanism to the case should be kept tight. Otherwise the calibration may be changed by the severe twisting which the gauge receives when it is being attached to the tester. This stress may be avoided by the use of a union between the gauge and the tester. 
The 20-pound gauge should be calibrated by means of a mercury column. For higher pressures a gauge tester of the dead-weight type is convenient. The calibration is preferably made with the gauge in the position in which it is used and with the use of a valving device which will permit loading the gauge at approximately the same rate as in the bursting test. Corrections for the 20-pound gaige should be made to the nearest ounce, for the 80 -pound gauge to the nearest one-fourth pound, and for the 160-pound gauge to the nearest one-half pound. This corresponds to a sensitivity of 1 part in 320 for the maximum gauge reading.

\section{SIZE OF THE ORIFICE IN THE CLAMPING HEAD}

The percentage of elongation at rupture being constant for a given paper, the ratio of the diameter $C$, of the orifice to the length of the arc of paper subtending it at rupture is constant as $C$ varies, from which it follows that the angle $\theta$ subtended by the arc (and consequently its sine) is constant. Therefore, $R \sin \frac{\theta}{2}=\frac{C}{2}$, where $R$ is the radius of curvature. A constant relationship thus exists between $R$ and $C$ for a given paper at the moment of rupture, which is independent of the size of the orifice. Substituting this value of $R$ in equation (1), section $\mathrm{V}$, we have $P C=4 T \sin \frac{\theta}{2}$. Inasmuch as the tensile breaking strength $T$ of a given paper is constant, it follows that the product of the bursting pressure $P$ and orifice diameter $C$ is constant. Foster ${ }^{4}$ showed this to be approximately true for textiles by using three testers having bursting orifices $1 \frac{1}{4}, 4$, and 8 inches in diameter. Some data obtained by Abrams ${ }^{5}$ also tend to substantiate this relation. If in Table 7 of his report the bursting pressures are multiplied by the square roots of the respective orifice areas, the spread of the products is only about 8 per cent. Abrams did not, like Foster, use different testers having bursting orifices of different sizes, but used washers with different sizes of holes in the center through which the paper was forced. This procedure allows the paper to stretch over a larger area than that corresponding to the bursting orifice, as a consequence of which the ratio of the radius of curvature to the diameter of the bursting orifice is no longer constant for a given material. The smaller the hole in the washer the more is the bursting pressure increased over what it would be if the paper were free to stretch only over the area corresponding to the bursting orifice. Hence, the product of orifice diameter and bursting pressure obtained in this manner, instead of being constant, should be somewhat larger for the smaller orifice diameters than for the larger ones. This is true of Abrams's data. Table 4 gives the results of additional tests by the authors. Measurements were made on five samples of paper, each recorded result being the average of 20 individual tests. A set of metal plates was used having orifice diameters approximately as follows: $1 / 4,1 \frac{1}{2}, 3 / 4,1,1 \frac{1}{4}, 1 \frac{31}{8}$, and 2 inches. The values for the product of bursting pressures and orifice diameters in the last column of Table 4 are greatest for the smallest diameter and decrease uniformly as the orifice is increased in size, as one might expect. 
TABLE 4.-Effect of size of bursting orifice on bursting pressure

[Clamped area constant except for the two largest orifices]

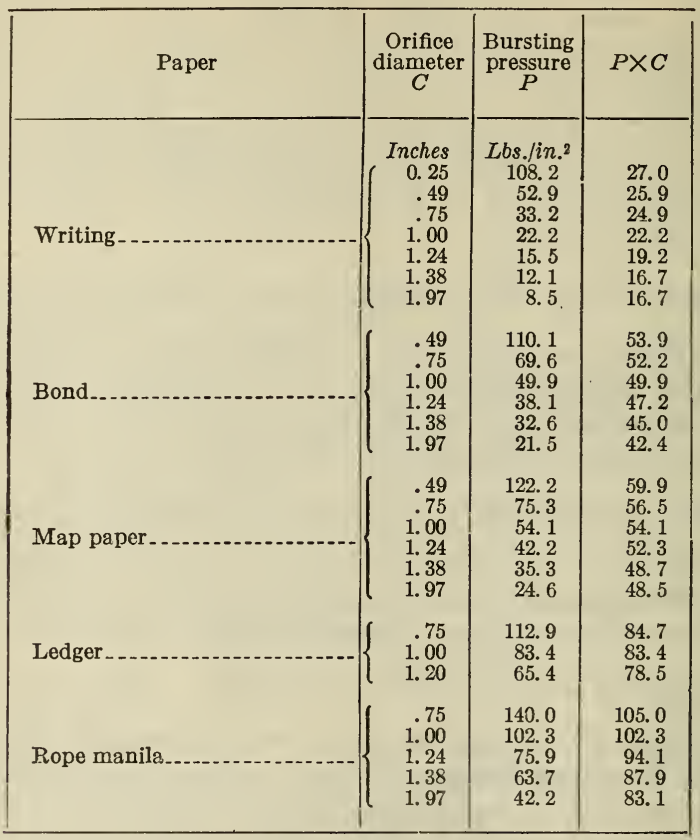

When it was first proposed to replace the rubber-ring type of clamping head with an all-metal one there was inadvertently introduced into the experiments the effect of a small difference in the size of the bursting orifice. The orifice in the rubber ring is reamed to a

TABLE 5.-Comparison of rubber and metal bursting orifices

\begin{tabular}{|c|c|c|c|c|}
\hline \multirow{3}{*}{ Paper } & \multicolumn{4}{|c|}{ Bursting pressure } \\
\hline & \multirow{2}{*}{$\begin{array}{l}\text { Rubber } \\
\text { orifice }^{1}\end{array}$} & \multicolumn{3}{|c|}{ Metal orifices } \\
\hline & & $\begin{array}{c}1.20 \\
\text { inches } \\
\text { diameter }\end{array}$ & $\begin{array}{c}1.24 \\
\text { inches } \\
\text { diameter }\end{array}$ & $\begin{array}{l}\text { Differ- } \\
\text { ence }\end{array}$ \\
\hline & Lbs./in. ${ }^{2}$ & Lbs./in.? & Lbs./in. ${ }^{2}$ & Per cent \\
\hline Writing-- & $\begin{array}{l}16.6 \\
\end{array}$ & 16.5 & 16. 3 & 1.2 \\
\hline Bond. & $\left\{\begin{array}{l}39.9 \\
60.7\end{array}\right.$ & 61.4 & $\begin{array}{l}51.9 \\
59.3\end{array}$ & $\begin{array}{l}2.8 \\
3.4\end{array}$ \\
\hline Ledger. & 53.8 & 53.2 & 52.7 & 0.9 \\
\hline Kraft. . . . & $\begin{array}{l}67.2 \\
63.1\end{array}$ & $\begin{array}{l}65.9 \\
59.9\end{array}$ & $\begin{array}{l}65.1 \\
59.0\end{array}$ & $\begin{array}{l}1.2 \\
1.5\end{array}$ \\
\hline Rope, manila & 79.7 & 78.8 & 77.0 & 2.3 \\
\hline
\end{tabular}

1 The nominal diameter of the rubber orifice was 1.24 inches, but under clamping pressure the orifico closed up to an actual diameter of about 1.20 inches. 
diameter of 1.24 inches. The first all-metal clamping heads experimented with were, therefore, made with orifices 1.24 inches in diameter. When comparative tests were made of the two types of clamps it was found that the all-metal clamp gave somewhat lower bursting values than the rubber-ring type of clamp. A new rubber-ring typo of clamping head was placed over a diaphragm plate and arranged in a hydraulic press in such a manner that measurements of the orifice diameter could be made. When the normal clamping load was applied the orifice diameter was found to be only 1.20 inches, which is the actual working diameter. An all-metal clamping head having an orifice 1.20 inches in diameter gave results in satisfactory agreement with the rubber ring. Comparative tests of the rubber and metal orifice plates are shown in Table 5 , each value tabulated being the average of from 40 to 100 individual bursting tests. The difference in results obtained with the two all-metal clamping heads having orifices of 1.20 and 1.24 inches in diameter ranged from 0.9 to 3.4 per cent, the theoretical difference being 3.2 per cent.

\section{COMPARISON OF THE NEW, MOTOR-DRIVEN TESTERS WITH THE OLDER, HAND-DRIVEN TESTERS}

The hand-driven bursting testers, which have been in use for a long time, are being replaced to a considerable extent by a new motor-driven model having the all-metal clamping head. Tho motor-driven testers at first had a highor operating speed than that prescribed in the official bursting-test method, a condition which has since been corrected. Some comparative tests were therefore made with this new high-speed tester and one of the older hand-driven testers equipped with the rubber orifice plate and operated at normal speed. The results of these tests are shown in Table 6 . Data were also taken from routine test records of such tests as had been made on the two testers. These results, which are averages of only 10 test in each case, are shown in Table 7 . In most cases the agreement is about as good as is ordinarily obtained in duplicate tests on the same tester.

TABLE 6.-Comparison of (a) new motor-driven tester having metal clamping healb containing the 1.20-inch orifice with (b) the regular hand-driven tester with rubber orifice plate

\begin{tabular}{|c|c|c|}
\hline \multirow{2}{*}{ Paper } & \multicolumn{2}{|c|}{ Bursting pressure } \\
\hline & (a) & (b) \\
\hline $\begin{array}{l}\text { Writing-.. } \\
\text { Bond } \\
\text { Ledger-.-. } \\
\text { Bond } \\
\text { Kraft..... } \\
\text { Ledger_... } \\
\text { Rope, manila.... }\end{array}$ & $\begin{array}{c}\text { Lbs./in. }{ }^{2} \\
16.1 \\
39.1 \\
52.1 \\
58.2 \\
59.2 \\
64.8 \\
77.1\end{array}$ & $\begin{array}{r}\text { Lbs./in.2 } \\
16.3 \\
38.9 \\
53.5 \\
59.4 \\
61.6 \\
67.0 \\
78.7\end{array}$ \\
\hline
\end{tabular}


TABLE 7.-Same as Table 6, the data being taken from the records of regular routine tests

\begin{tabular}{|c|c|c|}
\hline \multirow{2}{*}{ Paper } & \multicolumn{2}{|c|}{ Bursting pressure } \\
\hline & (a) & (b) \\
\hline $\begin{array}{l}\text { Label_-. } \\
\text { Index curds-- }\end{array}$ & $\begin{array}{c}\text { Lbs./in. }{ }^{2} \\
47.9\end{array}$ & $\begin{array}{c}L b s . / i n . .^{2} \\
48.6\end{array}$ \\
\hline Ledger... & $\begin{array}{l}64.3 \\
64.3\end{array}$ & $\begin{array}{l}63.4 \\
63.3\end{array}$ \\
\hline $\begin{array}{l}\text { Bristol board } \\
\text { Test liner }\end{array}$ & $\begin{array}{r}142.8 \\
96.7\end{array}$ & $\begin{array}{r}143.9 \\
97.2\end{array}$ \\
\hline
\end{tabular}

\section{RELATION OF BURSTING TO TENSILE RESULTS}

\section{THEORY}

The bursting test is commonly regarded as one of the cardinal tests of paper and is not ordinarily thought of as being encompassed by other tests commonly made on paper. There is, however, a wellknown formula suggestive of a definite relation between the so-called bursting strength and the tensile properties of paper as ordinarily determined with the tensile tester. This equation-

$$
P R=2 T
$$

in which $P$ is the internal pressure, $R$ the radius of curvature, and $T$ the tensile stress per unit width, relates the pressure within a spherical shell to the tensile stress per unit width of the material composing the spherical shell. Some years ago one of the authors called attention to this equation and suggested its applicability to the bursting test of paper. ${ }^{6}$ There are, however, several circumstances which foster doubt as to the validity of applying this equation to the bursting test of paper, as a consequence of which no serious attempt appears to have been made heretofore to test its validity in the field of paper, although Foster ${ }^{7}$ in a study of textile materials found a fairly good agreement between the experimental tensile results and those calculated by means of this equation from data obtained with the bursting tester.

If paper were an isotropic material, and if a spherical surface were known to be formed at the instant of rupture in the bursting test, it would obviously be valid to apply the equation to the bursting test. The internal pressure is directly indicated on a maximum-reading gauge and the paper gives way after the manner of a tensile rupture. There are, however, some reasons for doubting the sphericity of the paper surface. In the ideal case of a homogeneous, isotropic material a spherical surface would not be developed from a plane sheet of material under pressure as in the bursting test, as has been shown by mathematieal analysis in the case of a plate held at the edges and subjected to uniform pressure. Moreover, paper is not an isotropic material. In the machine direction it is stronger, but has a smaller elongation at rupture than in the cross direction. It might be

- An Analysis of the Strength of Paper, Paper Trade J.; Mar. 20, 1924.
See footnoto 4, p. 345. 


\section{B. S. Journal of Research, RP278}

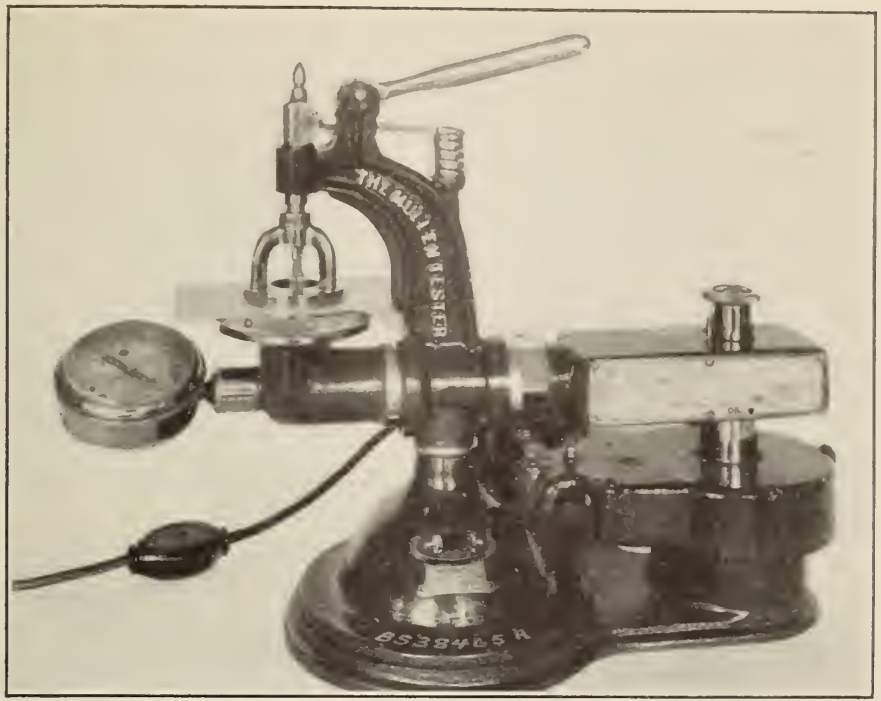

Figure 1.-Bursting strength tester for paper

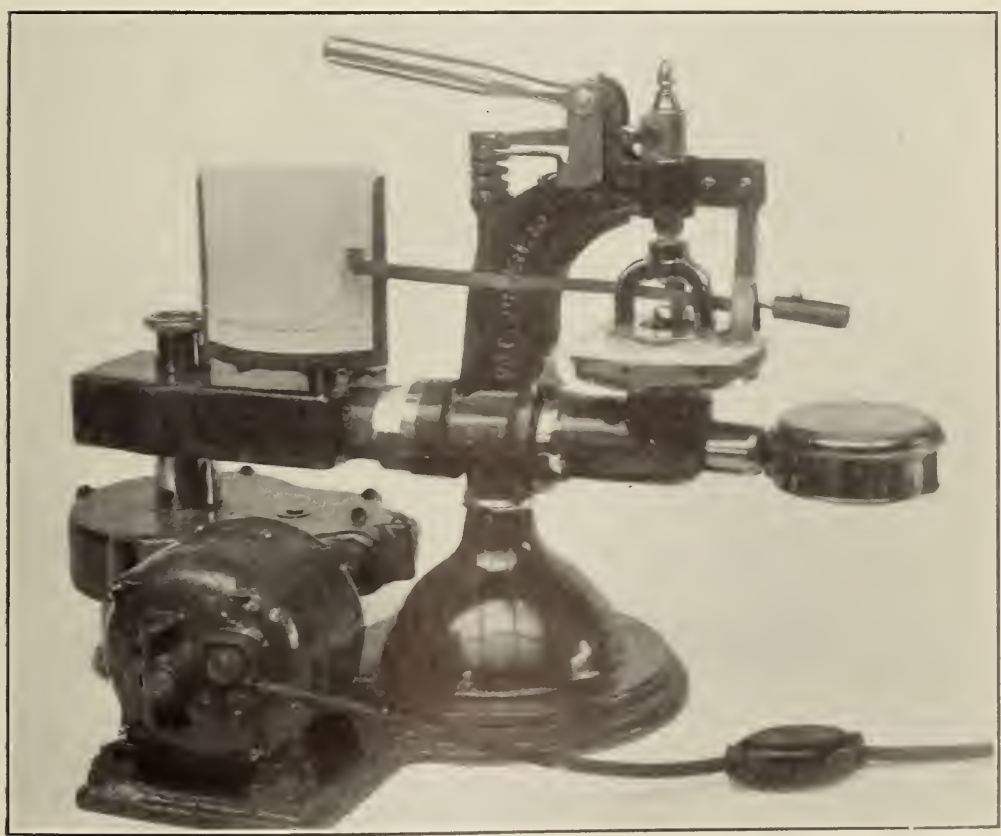

FIGURE 2.-Bursting tester with device altached for measuring the verticul deflection of specimen 
reasoned, therefore, that if the surface is not spherical in the ideal case it surely would not be in the case of an anisotropic material, such as paper under conditions far beyond the elastic limit of the material. On the other hand, it seems not improbable that the surface developed by paper, the thickness of which is very small in comparison with the length of the specimen and the stretch of which is in large measure permanent deformation, might approach a spherical surface.

In applying equation (1).T is taken as the tensile breaking strength per unit width in the machine direction of the paper, since the limit of stretch is first reached in this direction under the conditions of the bursting test. It is an experimental fact that in the bursting test the rupture begins with the failure of machine direction fibers. As a matter of fact the bursting test is often used as a means of determining the machine direction of paper.

The value of $R$, the radius of curvature, is calculated from the rise of the apex of the stressed specimen by the following equation:

$$
R=\frac{4 V^{2}+C^{2}}{8 V}
$$

in which $R$ is the radius of curvature, $V$ the vertical rise of the paper at the apex, and $C$ the diameter of the bursting orifice.

In order to relate the elongation at rupture to the radius of curvature, one other equation is necessary:

$$
E=\frac{200 R}{C} \arcsin \frac{C}{2 R}-100
$$

in which $E$ is the elongation at rupture in per cent, $R$ is the radius of curvature, and $C$ is the diameter of the bursting orifice. The value of $C$ for the bursting tester used in these experiments is 1.20 inches.

\section{SPECIAL APPARATUS}

The value of $V$, the vertical rise of the paper in the bursting test, is small, only one or two tenths of an inch. This deflection is measured by the special recorder illustrated in Figure 2. It consists of a lever of the third class, the shorter arm being actuated by the rise of the paper and the longer arm being provided with a recording pen which makes an autographic record on a chart moved horizontally by the pressure plunger of the bursting tester. When the paper bursts a sharp break occurs in the curve on the chart, from which the vertical rise of the pen corresponding to the position of rupture can be measured. The apparatus was calibrated by means of a dial micrometer. It was found that the clamping pressure squeezed the paper into the bursting orifice enough to cause a slight initial depression of the recording pen of about 1 or $2 \mathrm{~mm}$ which had to be taken into account in determining the value of $V$ from the graphs. A typical autographic deflection curve is shown in Figure 3.

\section{EXPERIMENTAL DATA}

Tests were made of bursting pressure and vertical deflection on seven samples of paper, and simultaneously tests were also made of tensile breaking strength and elongation at rupture with the tensile 
tester. Ten tests were made on each sample with each tester. Precautions were taken to eliminate errors as far as possible. The samples were conditioned and the tests were made under standard hygrometric conditions. Each sample was carried through all the tests before the next sample was tested. In making the tensile tests,

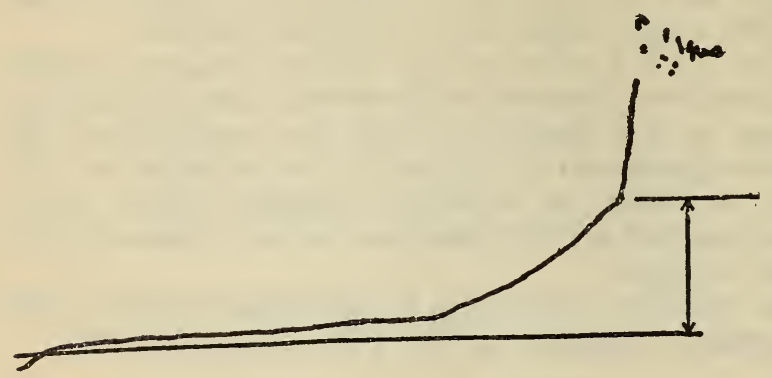

FIgURE 3.-Typical autographic deflection curve for paper being burst

The interval indicated by the arrow represents the vertical movement of the recording pen up to the instant of rupture.

specimens $180 \mathrm{~mm}$ in length were used so that the elongation data could be read directly in percentage. The results were observed immediately after the specimens broke, as it was found that if one waited until the stressing jaw was returned to its initial position the vibration would often cause the elongation pointer to indicate too great a reading. The tensile tester was calibrated over the necessary range before the tests were made. The bursting tests were made with the all-metal tripod clamp having a bursting orifice 1.20 inches in diameter. The clamping spring was calibrated and a clamping pressure applied sufficient to prevent slipping.

With the results thus obtained, it was possible to calculate by means of the three equations of the preceding section the machine

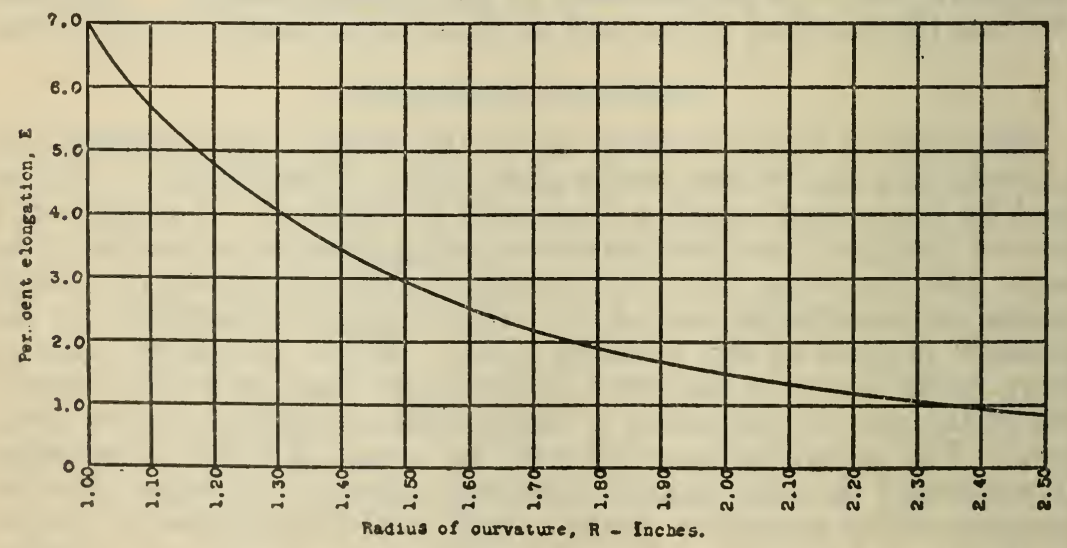

FIGURE 4.-Graph of the equation, $E=\frac{200 R}{C}$ arc sin $\frac{C}{2 R}-100$, in which $C=1.20$ inches

direction tensile breaking strength from the bursting data, or the "bursting strength" from the tensile data, or the elongation at rupture (machine direction) from the bursting pressure and the tensile breaking strength (machine direction). In order to determine $R$ from a known value of $E$ when solving equation (1) for $P$ 
or $T$, it was necessary to employ a graphic solution of equation (3) between the necessary limits, since $R$ can not be expressed explicitly in terms of $E$. The graph of this equation is shown in Figure 4, from which either $E$ or $R$ may be determined if the other is known. Both the experimental and the calculated values, arrangeil for ready comparison, are shown in Table 8.

For the convenience of the reader the calculated quantities in this table are, as far as possible, expressed explicitly in terms of the experimental quantities in the following four equations, which are obtained by combination of equations (1), (2), and (3):

TABLE 8.-Relation of bursting and tensile results

\begin{tabular}{|c|c|c|c|c|c|c|c|c|c|c|}
\hline Kind of paper & \begin{tabular}{|} 
Tensile \\
break- \\
ing \\
strength, \\
ma- \\
chine \\
di- \\
rection \\
\\
$T$
\end{tabular} & $\begin{array}{c}\text { Calcu- } \\
\text { lated } \\
\text { value of } \\
T \text { from } \\
P \text { and } V\end{array}$ & $\begin{array}{l}\text { Tensile } \\
\text { break- } \\
\text { ing } \\
\text { strength, } \\
\text { cross di- } \\
\text { rection }\end{array}$ & $\begin{array}{l}\text { Burst- } \\
\text { ing } \\
\text { pres- } \\
\text { sure }\end{array}$ & $\begin{array}{c}\text { Calcu- } \\
\text { lated } \\
\text { value of } \\
P \text { from } \\
T \text { and } E\end{array}$ & $\begin{array}{c}\text { Ver- } \\
\text { tical } \\
\text { deflec- } \\
\text { tion in } \\
\text { burst- } \\
\text { ing } \\
\text { test } \\
\\
\\
V\end{array}$ & $\begin{array}{c}\text { Elon- } \\
\text { gation } \\
\text { at rup- } \\
\text { ture, } \\
\text { ma- } \\
\text { chine } \\
\text { direc- } \\
\text { tion } \\
\text { deter- } \\
\text { mined } \\
\text { from } V \\
\\
E_{b}\end{array}$ & $\begin{array}{c}\text { Elon- } \\
\text { gation } \\
\text { at rup- } \\
\text { ture, } \\
\text { ma- } \\
\text { chine } \\
\text { direc- } \\
\text { tion } \\
\text { from } \\
\text { tensile } \\
\text { test } \\
E\end{array}$ & $\begin{array}{c}\text { Elon- } \\
\text { gation } \\
\text { at rup- } \\
\text { ture, } \\
\text { ma- } \\
\text { chine } \\
\text { direc- } \\
\text { tion } \\
\text { calcu- } \\
\text { lated } \\
\text { from } P \\
\text { and } T \\
E_{s}\end{array}$ & $\begin{array}{l}\text { Elon- } \\
\text { gation } \\
\text { at rup- } \\
\text { ture, } \\
\text { cross } \\
\text { di- } \\
\text { rection }\end{array}$ \\
\hline 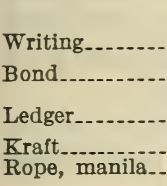 & $\begin{array}{l}\text { Lbs./in. } \\
18.3 \\
33.5 \\
43.2 \\
35.4 \\
51.4 \\
45.4 \\
67.4\end{array}$ & $\begin{array}{r}\text { Lbs./in. } \\
-17.6 \\
29.3 \\
38.5 \\
36.8 \\
50.9 \\
45.8 \\
65.9\end{array}$ & $\begin{array}{r}\text { Lbs./in. } \\
9.3 \\
17.5 \\
22.4 \\
22.4 \\
30.9 \\
21.2 \\
18.6\end{array}$ & $\begin{array}{r}\text { Lbs./in. }{ }^{2} \\
17.7 \\
39.3 \\
59.3 \\
53.3 \\
64.4 \\
55.8 \\
85.0\end{array}$ & \begin{tabular}{|c|} 
Lbs./in. ${ }^{2}$ \\
16.1 \\
45.9 \\
69.1 \\
53.3 \\
68.5 \\
55.7 \\
89.9
\end{tabular} & $\begin{array}{l}\text { Inch } \\
0.093 \\
.126 \\
.147 \\
.137 \\
.118 \\
.114 \\
.121\end{array}$ & \begin{tabular}{|c} 
Per cent \\
1.5 \\
3.0 \\
4.0 \\
3.5 \\
2.6 \\
2.4 \\
2.7
\end{tabular} & \begin{tabular}{|c} 
Per cent \\
1.1 \\
3.1 \\
4.4 \\
3.8 \\
2.9 \\
2.4 \\
2.9
\end{tabular} & \begin{tabular}{|c} 
Per cent \\
1.4 \\
2.1 \\
3.1 \\
3.8 \\
2.5 \\
2.4 \\
2.6
\end{tabular} & $\begin{array}{r}\text { Per cent } \\
3.7 \\
5.8 \\
7.9 \\
5.7 \\
6.4 \\
5.7 \\
7.8\end{array}$ \\
\hline
\end{tabular}

$$
\begin{aligned}
& T_{b}=\frac{P R}{2}=\frac{P}{2} \frac{4 V^{2}+C^{2}}{8 V}=\frac{P}{4}\left(V+\frac{0.36}{V}\right) \\
& \begin{aligned}
E_{b}=\frac{200 R}{C} \arcsin \frac{C}{2 R}-100 \\
\quad=83.33\left(V+\frac{0.36}{V}\right) \arcsin \frac{1.2 V}{V^{2}-0.36}-100
\end{aligned} \\
& E_{s}=\frac{200}{C} \cdot \frac{2 T}{P} \arcsin \frac{C}{2} \cdot \frac{P}{2 T}-100=\frac{10^{3} T}{3 P} \arcsin \frac{0.3 P}{T}-100 \\
& P_{t}=\frac{2 T}{R}=\frac{2 T}{f(E)}
\end{aligned}
$$

In equation (7) the value of $R$ is determined from Figure 4, which is the graph of equation (3). The angles involved in equations (5) and $(6)$ are in radians.

Although there are a few instances in the table in which the agreement between experimental and calculated values of properties is not all that might be desired, it is, in general, as good as that of duplicate experimental determinations.

From these results it is evident that the bursting strength is determined by the properties of paper in its stronger direction and that it 
is therefore inadequate for the specification of the strength of certain types of paper. A typical case in point is the specification of strength, to be determined by means of the bursting test, of rather poorly formed perforated papers in rolls, such as toilet tissue and paper towels. In many cases such paper will not tear consistently along the perforations, but splits lengthwise because of the disparity between the machine direction strength and the cross-direction strength, a condition about which the bursting test can give no information. From the viewpoint of the testing laboratory the bursting test appears to be merely a convenient, although a somewhat inadequate, substitute for the tensile test. Its justification appears to be chiefly in the mobility and sturdiness of the testing device and the facility with which a direct determination may be made of an empirica quantity, involving strength and stretch. The bursting test is regarded by the paper industry as having considerable significance in connection with many uses of paper, and appears to be especially valuable to the practical paper maker in mill control.

\section{CONCLUSIONS}

1. Slipping of the specimen during the test gives high bursting values. A clamping force of about 1,000 pounds is required to insure against the slipping of the common types of flexible papers. Slipping is readily detected by the movement of the edges of the specimen during the test. Within certain limits the gripping efficiency is practically independent of the area of the clamping surfaces.

2. All-metal clamps are superior in clamping efficiency to the rubber ring type, as well as being permanent in respect to the size and shape of the bursting orifice.

3. The bursting strength of paper increases slightly with the testing rate. For a constant plunger speed the testing rate is decreased by the presence of air in the hydraulic chamber. The bursting value may be affected by poor adjustment of the damping spring in the pressure gauge, the error being greater the higher the testing rate and the greater the gauge reading. The testing rate may conveniently be expressed in terms of the time interval of stressing the specimen and in order to correspond to the officially designated rate of testing, this should be about 1 second for papers bursting between 50 and 75 pounds per square inch.

4. The diaphragm error in the type of tester studied is small, in consequence of which the manner of installing the diaphragm is a negligible factor in the results.

5. The viscosity of the gylcerine is also a negligible factor in the bursting results.

6. As much friction as is consistent with the ready and smooth response of the gauge appears to be the optimum adjustment of the damping spring in the pressure gauge.

7. For a given paper the bursting pressure is approximately inversely proportional to the diameter of the bursting orifice. The all-metal clamping head with an orifice 1.20 inches in diameter is substantially the equivalent in performace of the old-style rubber faced clamping head, the orifice of which has a nominal diameter of 1.24 inches but an effective diameter of only 1.20 inches. 
8. The bursting strength of paper is determined by the tensile properties of the machine direction of paper, is apparently not affected by the cross direction properties, and reveals nothing which could not be determined by a tensile test. Of the three properties, bursting strength, tensile breaking strength (machine direction), and elongation at rupture (machine direction), any one may be readily calculated if the other two are known. Because of this relation it is possible to supply to some extent missing elongation data in the literature of paper testing.

Washington, December 19, 1930. 